\title{
ON SOME CLASSES OF DEMICOMPACT LINEAR RELATION AND SOME RESULTS OF ESSENTIAL PSEUDOSPECTRA
}

\begin{abstract}
A. Ammar, A. Jeribi, B. Saadaoui. On some classes of demicompact linear relation and some results of essential pseudospectra, Mat. Stud. 52 (2019), 195-210.

The main goal of this paper is to give some perturbation results and some relations between the essential pseudospectra of the sum of two multivalued linear operator and the essential pseudospectra of each of these multivalued linear operator.
\end{abstract}

1. Introduction. Spectral theory is an important topic of functional analysis. It has numerous applications in many parts of mathematics and physics including matrix theory, complex analysis, differential and integral equations, control theory and quantum physics. In recent years, spectral theory has witnessed an explosive development.

There are many types of spectra, both for one or serval commuting operators, with important applications, for example the approximate points spectrum, Taylor spectrum, essential spectrum, etc.

Recently, the concept of demicompactness with respect to a closed densely defined linear operator, as a generalization of the class of demicompact operator introduced by Petryshyn in [18]. This description involved the use of notions that were originally developed for demicompactness in linear spaces by W. Chaker, A. Jeribi, and B. Krichen [11] and systematically treated in the context of the bundle operator in [16] by B. Krichen. Lately, in [9] A. Ammar, H. Daoud and A. Jeribi defined the demicompact of a linear relation by $T: \mathcal{D}(T) \subseteq X \rightarrow X$ is said to be demicompact if for every bounded sequence $\left\{x_{n}\right\}$ in $\mathcal{D}(T)$ such that $Q_{I-T}(I-T) x_{n} \rightarrow y \in X / \overline{(I-T)(0)}$, there is a convergent subsequence of $\left\{Q_{T} x_{n}\right\}$. Then, we generalize some results given in [11] to multivalued linear operators. An important direction we are concerned with the extension of the concept of pseudospectra. In [21] J. M. Varah introduced the first idea of pseudospectra. In [22] J. H. Wilkinson came with the modern interpretation of pseudospectrum where he defined it for an arbitrary matrix norm induced by a vector norm and has been employed by other authors $[15,17,19,20]$. In $[9,10]$, A. Ammar, H. Daoud and A. Jeribi defined the pseudospectra of a linear relation $T$ by

$$
\sigma_{\varepsilon}(T):=\sigma(T) \cup\left\{\lambda \in \mathbb{C}:\left\|(\lambda-T)^{-1}\right\|>\frac{1}{\varepsilon}\right\},
$$

where $\varepsilon>0$ and $\sigma(T)$ is the spectrum of linear relation $T$. By convention we write $\|(\lambda-$ $T)^{-1} \|=\infty$ if $(\lambda-T)^{-1}$ is unbounded or nonexistent, i.e., if $\lambda$ is in $\sigma(T)$, and we define the 
notion of Weyl pseudospectra of a closed linear relation in a Banach space by:

$$
\sigma_{w, \varepsilon}(T):=\bigcap_{K \in \mathcal{K}_{T}(X)} \sigma_{\varepsilon}(T+K)
$$

where $\mathcal{K}_{T}(X):=\{K \in K \mathcal{R}(X): \mathcal{D}(K) \supset \mathcal{D}(T)$ and $K(0) \subset T(0)\}$.

In this paper, the symbols $X, Y$ stand for infinite dimensional Banach spaces over the same field $\mathbb{K}(\mathbb{K}$ being $\mathbb{R}$ or $\mathbb{C}$ ). A multivalued linear operator or linear relation is a mapping $T \subset X \times Y$ which goes from a subspace $\mathcal{D}(T) \subset X$ called the domain of $T$, into the collection of nonempty subsets of $Y$ such that $T\left(\alpha_{1} x_{1}+\alpha_{2} x_{2}\right)=\alpha_{1} T\left(x_{1}\right)+\alpha_{2} T\left(x_{2}\right)$ for all nonzero scalars $\alpha_{1}, \alpha_{2}$ with $x_{1}, x_{2} \in \mathcal{D}(T)$. For $x \in X \backslash \mathcal{D}(T)$ we define $T x=\varnothing$. With this convention, we have

$$
\mathcal{D}(T)=\{x \in X: T x \neq \varnothing\} .
$$

The collection of linear relations as defined above will be denoted by $L \mathcal{R}(X, Y)$. A linear relation $T \in L \mathcal{R}(X, Y)$ is uniquely determined by and identified with its graph, $G(T)$, defined by

$$
G(T)=\{(x, y) \in X \times Y: x \in \mathcal{D}(T), y \in T x\} .
$$

The inverse of $T \in L \mathcal{R}(X, Y)$ is the linear relation $T^{-1}$ defined by

$$
G\left(T^{-1}\right)=\{(y, x) \in Y \times X: \quad(x, y) \in G(T)\} .
$$

Let $T, S \in L \mathcal{R}(X, Y)$. Then their algebraic sum $T+S$ is also a linear relation defined by $G(T+S):=\{(x, u+v):(x, u) \in G(T),(x, v) \in G(S)\}$. Similarly, if $T \in L \mathcal{R}(X, Y)$ and $S \in L \mathcal{R}(Y, Z)$, then their product $S T$ is also a linear relation defined by

$$
G(S T):=\{(x, z) \in X \times Z:(x, y) \in G(T) \text { and }(y, z) \in G(S) \text { for some } y \in Y\} .
$$

If $M$ is a subspace of $X$ such that $M \cap \mathcal{D}(T) \neq \varnothing$, then $T_{\mid M \cap \mathcal{D}(T)}:=T_{\mid M}$ is defined by

$$
G\left(T_{\mid M}\right):=\{(x, y) \in G(T): x \in M\} \text {. }
$$

The quotient map from $Y$ onto $Y / \overline{T(0)}$ is denoted by $Q_{T}$. It is easy to see that $Q_{T} T$ is single valued so that we can define

$$
\|T x\|:=\left\|Q_{T} T x\right\| \text { for all } x \in \mathcal{D}(T) \text { and }\|T\|:=\left\|Q_{T} T\right\| .
$$

Let $T \in L \mathcal{R}(X, Y)$. The range of $T$ is the subspace $R(T):=\{y:(x, y) \in G(T)\}$ and $T$ is called surjective if $R(T)=Y$. The subspace $T^{-1}(0)$ is denoted by $N(T)$ and $T$ is called injective if $N(T)=\{0\}$. If $T$ is both injective and surjective, then we say that $T$ is bijective. Observe that $T x=y+T(0)$, for any $y \in T x$.

We say that $T \in L \mathcal{R}(X, Y)$ is continuous if $\|T\|<\infty$; bounded if it is continuous and $\mathcal{D}(T)=X$; open if $T^{-1}$ is continuous; equivalent if its minimum modulus $\gamma(T)$ is a positive number, where

$$
\gamma(T):=\sup \{\lambda \geq 0: \lambda d(x, N(T)) \leq\|T x\|, \quad x \in \mathcal{D}(T)\},
$$

where $d(x, N(T))$ the distance between $x$ and $N(T)$. A linear relation $T$ is said to be closed if its graph is closed. Similarly, $T$ is called closable if $\bar{T}$ is an extension of $T$ where the closure of $T, \bar{T}$, is defined by $G(\bar{T}):=\overline{G(T)}$. We denote the class of all bounded linear relations from 
$X$ to $Y$ by $B \mathcal{R}(X, Y)$. The collection of all closed linear relations from $X$ to $Y$ is denoted by $C \mathcal{R}(X, Y)$. We denote the class of compact linear relations from $X$ to $Y$ by $K \mathcal{R}(X, Y)$. For $T \in L \mathcal{R}(X, Y)$, we write $\alpha(T):=\operatorname{dim} N(T), \beta(T):=\operatorname{dim} Y / R(T), \bar{\beta}(T):=\operatorname{dim} Y / \overline{R(T)}$ and the index of $T$ is the quantity $i(T):=\alpha(T)-\beta(T)$ provided that $\alpha(T)$ and $\beta(T)$ are not both infinite.

We say $T$ is upper semi-Fredholm, if there exists a finite codimensional subspace $M$ of $\mathcal{D}(T)$ for which $T_{/ M}$ is injective and open (this is equivalent to the corresponding property for $\left.Q_{T} T\right)$. $T$ is called lower semi-Fredholm if $T^{\prime}$ is upper semi-Fredholm. If $T$ is both upper semi-Fredholm and lower semi-Fredholm, we say that $T$ is Fredholm. We denote the class of upper semi-Fredholm, lower semi-Fredholm and Fredholm linear relations from $X$ into $Y$ by $\mathcal{F}_{+}(X, Y), \mathcal{F}_{-}(X, Y)$ and $\mathcal{F}(X, Y)$, respectively. If $X=Y$ then $\mathcal{F}_{+}(X, Y), \mathcal{F}_{-}(X, Y)$, $\mathcal{F}_{ \pm}(X, Y)$ and $\mathcal{F}(X, Y)$ are replaced by $\mathcal{F}_{+}(X), \mathcal{F}_{-}(X), \mathcal{F}_{ \pm}(X)$ and $\mathcal{F}(X)$, respectively.

A closed linear relation $T$ acts from a Banach space $X$ into $Y$. The set of upper semiFredholm relations is defined by:

$$
\Phi_{+}(X, Y)=\{T \in \mathcal{C}(X, Y): \alpha(T)<\infty \text { and } R(T) \text { is closed in } Y\},
$$

and the set of lower semi-Fredholm relations is defined by:

$$
\Phi_{-}(X, Y)=\{T \in \mathcal{C}(X, Y): \beta(T)<\infty \text { and } R(T) \text { is closed in } Y\} .
$$

$\Phi(X, Y):=\Phi_{+}(X, Y) \cap \Phi_{-}(X, Y)$ denotes the set of Fredholm relations from $X$ into $Y$ and $\Phi_{ \pm}(X, Y):=\Phi_{+}(X, Y) \cup \Phi_{-}(X, Y)$ denotes the set of semi-Fredholm relations from $X$ into $Y$. If $X=Y$ then $\Phi_{+}(X, Y), \Phi_{-}(X, Y), \Phi_{ \pm}(X, Y)$ and $\Phi(X, Y)$ are replaced by $\Phi_{+}(X)$, $\Phi_{-}(X), \Phi_{ \pm}(X)$ and $\Phi(X)$, respectively. The paper is organized as follows. Section 2 contains preliminary and auxiliary properties that we will need to prove the main results about linear relations in Banach spaces. In the last section we establish some perturbation results for essential spectra.

\section{Preliminaries.}

Lemma 1 ([13]). Let $X$ and $Y$ be two vector spaces and let $T \in L \mathcal{R}(X, Y)$. Then

(i) $\mathcal{D}\left(T^{-1}\right)=R(T)$ and $\mathcal{D}(T)=R\left(T^{-1}\right)$.

(ii) $T$ injective if and only if $T^{-1} T=I_{\mathcal{D}(T)}$.

(iii) $T$ is single valued if and only if $T(0)=\{0\}$.

(iv) $T T^{-1} y=y+T(0)$ and $T^{-1} T x=x+T^{-1}(0)$.

Lemma 2 ([3], Lemma 2.5). Let $S, T, A \in L \mathcal{R}(X, Y)$. If $S(0) \subset T(0)$ and $\mathcal{D}(T) \subset \mathcal{D}(S)$, then $T-S+S=T$.

Lemma 3 ([12], Lemma 2.4). Let $T \in \mathcal{L} \mathcal{R}(X, Y)$ and $S, R \in \mathcal{L} \mathcal{R}(Y, Z)$. If $T(0) \subset N(S)$ or $T(0) \subset N(R)$ then $(R+S) T=R T+S T$.

Proposition 1. Let $R, S, T \in L \mathcal{R}(X)$. Then,

(i) ([13, Proposition I.4.2(d)]) $(R+S) T \subset R T+S T$ with equality if $T$ is single valued.

(ii) ([13, Proposition I.4.2(e)]) $T(R+S)$ is an extension of $T R+T S$ and $T R+T S=T(R+S)$ if $\mathcal{D}(T)$ is the whole space. 
Proposition 2 ([5], Proposition 3.1). Let $S, T \in L \mathcal{R}(X)$ be such that $S(0) \subset T(0), \mathcal{D}(T) \subset$ $\mathcal{D}(S)$. If $T \in C \mathcal{R}(X)$ and $S$ is continuous, then $S+T \in C \mathcal{R}(X)$.

Lemma 4 ([14], Corollary 3.2). Let $S, T \in L \mathcal{R}(X)$. Suppose that $\mathcal{D}(S)=X$ and $T, S$ have finite indices. Then, $S T$ has a finite index and $i(S T)=i(S)+i(T)-\operatorname{dim}\left(T(0) \cap S^{-1}(0)\right)$.

Lemma 5 ([5], Theorem 2.2). Let $X$ be a Banach space and let $S, T \in C \mathcal{R}(X)$. Then

(i) $T \in \Phi_{+}(X)$ if and only if $Q_{T} T \in \Phi_{+}(X)$. In such case $i(T)=i\left(Q_{T} T\right)$.

(ii) $T \in \Phi_{-}(X)$ if and only if $Q_{T} T \in \Phi_{-}(X)$. In such case $i(T)=i\left(Q_{T} T\right)$.

(iii) If $S, T \in \Phi_{+}(X)$, then $S T \in \Phi_{+}(X)$ and $T S \in \Phi_{+}(X)$.

(iv) If $S, T \in \Phi_{-}(X)$ with $T S$ (respectively $S T$ ) closed, then $T S \in \Phi_{-}(X)$ (respectively $\left.S T \in \Phi_{-}(X)\right)$.

(v) If $S$ and $T$ are everywhere defined and $T S \in \Phi_{+}(X)$, then $S \in \Phi_{+}(X)$.

Definition 1 ([6], Definition 2.1). Let $S \in L \mathcal{R}(X, Y)$ be continuous.

(i) $S$ is called a Fredholm perturbation if $T+S \in \Phi(X, Y)$ whenever $T \in \Phi(X, Y)$ with $\operatorname{dim}(S(0))<\infty$ and $S(0) \subset T(0)$.

(ii) $S$ is called an upper semi-Fredholm perturbation if $T+S \in \Phi_{+}(X, Y)$ whenever $T \in$ $\Phi_{+}(X, Y)$ with $\operatorname{dim}(S(0))<\infty$ and $S(0) \subset T(0)$.

(iii) $S$ is called a lower semi-Fredholm perturbation if $T+S \in \Phi_{-}(X, Y)$ whenever $T \in$ $\Phi_{-}(X, Y)$ with $\operatorname{dim}(S(0))<\infty$ and $S(0) \subset T(0)$.

We denote by $\mathcal{P}(X)$ the set of Fredholm perturbations, by $\mathcal{P}_{+}(X)$ the set of upper semiFredholm perturbations, and by $\mathcal{P}_{-}(X)$ the set of lower semi-Fredholm perturbations.

Proposition 3 ([6], Proposition 2.2). Let $T \in L \mathcal{R}(X, Y)$ be closed and $S \in L \mathcal{R}(X, Y)$ be continuous. We have

(i) If $T \in \Phi_{+}(X, Y)$ and $S \in \mathcal{P}_{+}(X, Y)$, then $T+S \in \Phi_{+}(X, Y)$ and $i(T+S)=i(T)$.

(ii) If $T \in \Phi_{-}(X, Y)$ and $S \in \mathcal{P}_{-}(X, Y)$, then $T+S \in \Phi_{-}(X, Y)$ and $i(T+S)=i(T)$.

(iii) If $T \in \Phi(X, Y)$ and $S \in \mathcal{P}(X, Y)$, then $T+S \in \Phi(X, Y)$ and $i(T+S)=i(T)$.

Lemma 6 ([14], Corollary 3.2). Let $S, T \in L \mathcal{R}(X)$. Suppose that $\mathcal{D}(S)=X$ and $T, S$ have finite indices. Then, $S T$ has a finite index and $i(S T)=i(S)+i(T)-\operatorname{dim}\left(T(0) \cap S^{-1}(0)\right)$.

Theorem 1 ([13], Theorem V.10.3). Let $T \in L \mathcal{R}(X)$. Then the following are equivalent:

(i) $T \in \Phi_{+}(X)$;

(ii) There exists $A \in \mathcal{B}(X)$ and a finite rank projection $K$ such that $A T=I-K$.

3. Some perturbation results. In this section, we define the essential pseudospectra of a closed linear relation, study some properties and establish some results of perturbation on the context of linear relations.

Definition 2 ([7]). Let $\varepsilon>0$ and $T \in C \mathcal{R}(X)$. We define the essential pseudospectra of $T$ by:

$$
\sigma_{e 1, \varepsilon}(T):=\mathbb{C} \backslash\left\{\lambda \in \mathbb{C}: \lambda-T-S \in \Phi_{+}(X) \text {, for all } S \in \mathcal{J}_{T}(X)\right\}
$$




$$
\begin{array}{r}
\sigma_{e 2, \varepsilon}(T):=\mathbb{C} \backslash\left\{\lambda \in \mathbb{C}: \lambda-T-S \in \Phi_{-}(X), \text { for all } S \in \mathcal{J}_{T}(X)\right\}, \\
\sigma_{e 3, \varepsilon}(T):=\mathbb{C} \backslash\left\{\lambda \in \mathbb{C}: \lambda-T-S \in \Phi_{ \pm}(X), \text { for all } S \in \mathcal{J}_{T}(X)\right\}, \\
\sigma_{e 4, \varepsilon}(T):=\mathbb{C} \backslash\left\{\lambda \in \mathbb{C}: \lambda-T-S \in \Phi(X), \text { for all } S \in \mathcal{J}_{T}(X)\right\},
\end{array}
$$

where

$$
\mathcal{J}_{T}(X):=\{S \in L \mathcal{R}(X) \text { is continuous: }\|S\|<\varepsilon, S(0) \subset T(0) \text { and } \mathcal{D}(S) \supset \mathcal{D}(T)\} .
$$

Proposition 4. Let $T, S \in B \mathcal{R}(X)$ be such that $S(0) \subset T(0)$ and $\varepsilon>0$.

(i) If $D \in B \mathcal{R}(X)$ such that $\|D\|<\varepsilon, D(0) \subset S(0) \subset N(T)$ and $T(S+D) \in \mathcal{P}_{+}(X)$, then

$$
\sigma_{e 1, \varepsilon}(T+S) \subset \sigma_{e 1}(T) \cup \sigma_{e 1, \varepsilon}(S) .
$$

If, further, $(S+D) T \in \mathcal{P}_{+}(X)$ and $T(0) \subset N(S)$ or $T(0) \subset N(D)$ we get

$$
\sigma_{e 1, \varepsilon}(T+S)=\sigma_{e 1}(T) \cup \sigma_{e 1, \varepsilon}(S) .
$$

(ii) If $D \in B \mathcal{R}(X)$ such that $\|D\|<\varepsilon, D(0) \subset S(0) \subset N(T)$ and $T(S+D) \in \mathcal{P}_{+}(X)$, then

$$
\sigma_{e 2, \varepsilon}(T+S) \subset \sigma_{e 2}(T) \cup \sigma_{e 2, \varepsilon}(S) .
$$

If, further, $(S+D) T \in \mathcal{P}_{+}(X)$ and $T(0) \subset N(S)$ or $T(0) \subset N(D)$ we get

$$
\sigma_{e 2, \varepsilon}(T+S)=\sigma_{e 2}(T) \cup \sigma_{e 2, \varepsilon}(S) .
$$

(iii) If $D \in B \mathcal{R}(X)$ such that $\|D\|<\varepsilon, D(0) \subset S(0) \subset N(T)$ and $T(S+D) \in \mathcal{P}_{+}(X) \cap$ $\mathcal{P}_{-}(X)$, then

$$
\sigma_{e 3, \varepsilon}(T+S) \subset\left[\left(\sigma_{e 3}(T) \cup \sigma_{e 3, \varepsilon}(S)\right) \cup\left(\sigma_{e 3}(T) \cup \sigma_{e 2, \varepsilon}(S)\right) \cup\left(\sigma_{e 2}(T) \cup \sigma_{e 1, \varepsilon}(S)\right)\right] .
$$

If, further, $(S+D) T \in \mathcal{P}_{+}(X) \cap \mathcal{P}_{-}(X)$ and $T(0) \subset N(S)$ or $T(0) \subset N(D)$ we get

$$
\sigma_{e 3, \varepsilon}(T+S)=\left[\left(\sigma_{e 3}(T) \cup \sigma_{e 3, \varepsilon}(S)\right) \cup\left(\sigma_{e 3}(T) \cup \sigma_{e 2, \varepsilon}(S)\right) \cup\left(\sigma_{e 2}(T) \cup \sigma_{e 1, \varepsilon}(S)\right)\right] .
$$

(iv) If $D \in B \mathcal{R}(X)$ such that $\|D\|<\varepsilon, D(0) \subset S(0) \subset N(T)$ and $T(S+D) \in \mathcal{P}(X)$, then

$$
\sigma_{e 4, \varepsilon}(T+S) \subset \sigma_{e 4}(T) \cup \sigma_{e 4, \varepsilon}(S) .
$$

If, further, $(S+D) T \in \mathcal{P}(X)$ and $T(0) \subset N(S)$ or $T(0) \subset N(D)$ we get

$$
\sigma_{e 4, \varepsilon}(T+S)=\sigma_{e 4}(T) \cup \sigma_{e 4, \varepsilon}(S) .
$$

Proof. ( $i)$ Let $\lambda \in \mathbb{C}$. Since $\mathcal{D}(T)=X$, by Proposition 1 we have

$$
(\lambda-T)(\lambda-S-D)=\lambda(\lambda-S-D)-T(\lambda-S-D) .
$$

Since $\mathcal{D}(T)=X$, by using Proposition 1 the linear relation $(\lambda-T)(\lambda-S-D)$ can be written in the form

$$
(\lambda-T)(\lambda-S-D)=\lambda^{2}-\lambda S-\lambda D-\lambda T+T S+T D=\lambda(\lambda-S-D-T)+T(S-D) .
$$


Suppose that $\lambda \notin \sigma_{e 1}(T) \cup \sigma_{e 1, \varepsilon}(S)$, then $(\lambda-T) \in \Phi_{+}(X)$ and $\lambda-S-D \in \Phi_{+}(X)$ for all $D \in B \mathcal{R}(X)$ such that $\|D\|<\varepsilon$ and $D(0) \subset S(0)$ we deduce that $(\lambda-T)(\lambda-S-D) \in \Phi_{+}(X)$. When combined with the fact that

$$
T(S+D)(0)=T S(0) \subset T T^{-1}(0)=T(0)=(\lambda-S-D-T)(0)=T(0) .
$$

Applying Proposition 1, we have

$$
\begin{gathered}
(\lambda-T)(\lambda-S-D)+T(S-D)=\lambda(\lambda-S-D-T)-T(S-D)+T(S-D)= \\
=\lambda(\lambda-S-D-T) .
\end{gathered}
$$

In fact, $(\lambda-T)(\lambda-S-D) \in \Phi_{+}(X)$ and $T(S+D) \in \mathcal{P}_{+}(X)$. This implies that $\lambda-S-D-T \in$ $\Phi_{+}(X)$ for all $D \in B \mathcal{R}(X)$ such that $\|D\|<\varepsilon$ and $D(0) \subset S(0)$. Therefore, $\lambda \notin \sigma_{e 1, \varepsilon}(T+S)$,

$$
\sigma_{e 1, \varepsilon}(T+S) \subset \sigma_{e 1}(T) \cup \sigma_{e 1, \varepsilon}(S) .
$$

Conversely suppose that $\lambda \notin \sigma_{e 1, \varepsilon}(T+S)$, then $\lambda-S-D-T \in \Phi_{+}(X)$ for all $D \in B \mathcal{R}(X)$ such that $\|D\|<\varepsilon$ and $D(0) \subset S(0)$. Since $T, S, D \in B \mathcal{R}(X)$ by Proposition 1 we have

$$
(\lambda-S-D)(\lambda-T)=\lambda(\lambda-T)-S(\lambda-T)-D(\lambda-T)=\lambda^{2}-\lambda T-\lambda S+S T-\lambda D+D T \text {. }
$$

Since $T(0) \subset N(S)$ or $T(0) \subset N(D)$, by Lemma 3 we get

$$
(\lambda-S-D)(\lambda-T)=\lambda(\lambda-T-S-D)+(S+D) T .
$$

The relationships $(S+D) T \in \mathcal{P}_{+}(X)$ and $\lambda-T-S-D \in \Phi_{+}(X)$ yield $(\lambda-S-D)(\lambda-T) \in$ $\Phi_{+}(X)$. Likewise $T(S+D) \in \mathcal{P}_{+}(X)$ and $\lambda-T-S-D \in \Phi_{+}(X)$, then $(\lambda-T)(\lambda-S-D) \in$ $\Phi_{+}(X)$. By Theorem $5(i v)$, we get $(\lambda-T) \in \Phi_{+}(X)$ and $(\lambda-S-D) \in \Phi_{+}(X)$ for all $D \in B \mathcal{R}(X)$ such that $\|D\|<\varepsilon$ and $D(0) \subset S(0)$. Hence, $\lambda \notin \sigma_{e 1}(T) \cup \sigma_{e 1, \varepsilon}(S)$. We can deduce that

$$
\sigma_{e 1, \varepsilon}(T+S)=\sigma_{e 1}(T) \cup \sigma_{e 1, \varepsilon}(S) .
$$

(ii) This proof is similar to that of $(i)$.

(iii) By using the equalities $\sigma_{e 3}(T)=\sigma_{e 1}(T) \cap \sigma_{e 2}(T), \sigma_{e 3, \varepsilon}(S)=\sigma_{e 1, \varepsilon}(S) \cap \sigma_{e 2, \varepsilon}(S)$ and $\sigma_{e 3, \varepsilon}(T+S)=\sigma_{e 1, \varepsilon}(T+S) \cap \sigma_{e 2, \varepsilon}(T+S)$ from the assertions $(i)$ and (ii) the Proposition is proved.

(iv) Combining the assertion $(i)$ and $(i i)$.

Theorem 2. Let $T \in L R(X)$ such that $\operatorname{dim} T(0)<\infty$. If $T$ is demicompact, then $I-T \in$ $\Phi_{+}(X)$.

Theorem 3 ([8], Theorem 3.3). Let $T \in C \mathcal{R}(X)$ and $\operatorname{dim} T(0)<\infty$. If $\frac{1}{\mu} T$ is demicompact for each $\mu \in[1,+\infty[$, then $\mu-T \in \Phi(X)$.

Let $T \in C \mathcal{R}(X)$. We define the sets $\Lambda_{T}(X), \Upsilon_{T}(X)$ and $\Psi_{T}(X)$ by:

$$
\begin{aligned}
\Lambda_{T}(X) & =\{J \in L \mathcal{R}(X): \mu J \text { is demicompact for every } \mu \in[0,1]\}, \\
\Upsilon_{T, \varepsilon}(X) & =\left\{\begin{array}{cc}
K \in L \mathcal{R}(X): & \forall \mu \in \rho_{\varepsilon}(T+K), \mathcal{D}(T) \subset \mathcal{D}(K), \\
K(0) \subset T(0) \text { and } & \\
-(\mu-T-S-K)^{-1} K \in \Lambda_{T}(X) \forall S \in \mathcal{J}_{T}(X)
\end{array}\right\},
\end{aligned}
$$

We denote

$$
\sigma_{r, \varepsilon}(T):=\bigcap_{K \in \Upsilon_{T}(X)} \sigma_{\varepsilon}(T+K) .
$$


Theorem 4. Let $\varepsilon>0$ and $T \in L \mathcal{R}(X)$. Then

$$
\sigma_{e 4, \varepsilon}(T) \subset \sigma_{r, \varepsilon}(T) .
$$

Proof. Let $T \in C \mathcal{R}(X)$. Show that $\sigma_{e 4, \varepsilon}(T) \subset \sigma_{r, \varepsilon}(T)$. Indeed, let $\mu \notin \sigma_{r, \varepsilon}(T)$. Then $\mu \notin$ $\bigcap_{K \in \Upsilon_{T}(X)} \sigma_{\varepsilon}(T+K)$. Therefore, $\mu \in \cup_{K \in \Upsilon_{T}(X)} \rho_{\varepsilon}(T+K)$. Hence, there exists $K \in \Upsilon_{T}(X)$ such that $\mu \in \rho_{\varepsilon}(T+K)$. Furthermore, for all $S \in \mathcal{J}_{T}(X)$ we get $\mu \in \rho(T+K+S)$. We conclude that $-(\mu-T-S-K)^{-1} K$ is demicompact and $\mu-T-S-K$ is bijective. Hence, $\mu-T-K-S \in \Phi(X)$ and by applying Theorem 3.2, we get $I+(\mu-T-K-S)^{-1} K \in \Phi(X)$. Since $\mathcal{D}(T) \subset \mathcal{D}(S+K)$ and $S(0)+K(0) \subset T(0)$, by Lemma 2 we get

$$
\mu-T-S=\mu-T-S-K+K \text {. }
$$

Let $x \in \mathcal{D}(T)$. Since $\mathcal{D}(T)$ contains the ranges of $(\mu-T-S-K)^{-1} K$,

$$
\begin{gathered}
(\mu-T-S-K)\left(x+(\mu-T-S-K)^{-1} K x\right) \\
=(\mu-T-S-K) x+(\mu-T-S-K)(\mu-T-S-K)^{-1} K x .
\end{gathered}
$$

By Lemma $1(i v)$, we have $(\mu-T-S-K) x+(\mu-T-S-K)(\mu-T-S-K)^{-1} K x=$ $(\mu-T-S-K) x+K x+(\mu-T-S-K)(0)=(\mu-T-S-K) x+K x$. Therefore, $(\mu-T-S-K)\left(I+(\mu-T-S-K)^{-1} K\right)=\mu-T-S$. Hence, applying Theorem 5, we get $\mu-T-S \in \Phi(X)$ for all $S \in \mathcal{J}_{T}(X)$.

Corollary 1. Let $T \in C \mathcal{R}(X)$, and let $\mathcal{I}(X)$ be a subset of $\Upsilon_{T, \varepsilon}(X)\left(\right.$ resp. $\left.\Psi_{T, \varepsilon}(X)\right)$ containing $K \mathcal{R}(X)$. Then $\sigma_{e 4, \varepsilon}(T) \subset \bigcap_{K \in \mathcal{I}(X)} \sigma_{\varepsilon}(T+K)$.

Proof. Since $\mathcal{I}(X) \subset \Upsilon_{T, \varepsilon}(X)$. We infer that

$$
\bigcap_{K \in \Upsilon_{T, \varepsilon}(X)} \sigma_{\varepsilon}(T+K) \subset \bigcap_{K \in \mathcal{I}(X)} \sigma_{\varepsilon}(T+K) .
$$

It follows from Theorem 4 that $\sigma_{e 4, \varepsilon}(T) \subset \bigcap_{K \in \mathcal{I}(X)} \sigma_{\varepsilon}(T+K)$.

Theorem 5. Let $T, S \in B \mathcal{R}(X)$. If for every $\lambda \notin \sigma_{e 1, \varepsilon}(T)$ there exists $A_{\lambda}$ a left inverse modulo compact of $(\lambda-T-D)$ for all $D \in \mathcal{J}_{T}(X)$ such that $S A_{\lambda}$ is demicompact and $\operatorname{dim}\left(S A_{\lambda}(0)\right)<\infty$, then

$$
\sigma_{e 1, \varepsilon}(T+S) \subset \sigma_{e 1, \varepsilon}(T) .
$$

Proof. Let $\lambda \notin \sigma_{e 1, \varepsilon}(T)$. Then $\lambda-T-D \in \Phi_{+}(X)$ for all $D \in \mathcal{J}_{T}(X)$. By Theorem 1 , there exist $A_{\lambda} \in \mathcal{B}(X)$ and a finite rank projection $K$ such that $A_{\lambda}(\lambda-T-D)=I-K$. Since $S(0) \subset D(0)+T(0)=T(0)$ and $\mathcal{D}(S K)=\mathcal{D}(T)=X$, by Lemma 2 we have $\lambda-T-D-S=$ $\lambda-T-D-S+S K-S K$. By using Proposition 1 ( $i i)$, we get

$$
\begin{gathered}
\lambda-T-D-S+S K-S K=\lambda-T-D-S(I-K)-S K= \\
=\lambda-T-D-S A_{\lambda}(\lambda-T-D)-S K .
\end{gathered}
$$

Using Lemma 3 we get $\lambda-T-S-D=\left(I-S A_{\lambda}\right)(\lambda-T-D)-S K$. As $S A_{\lambda}$ is demicompact and $\operatorname{dim}\left(S A_{\lambda}(0)\right)<\infty$, it follows from Theorem 2 that $\left(I-S A_{\lambda}\right) \in \Phi_{+}(X)$ and we have $(\lambda-T-D) \in \Phi_{+}(X)$. Then by Theorem 5 we get $\left(I-S A_{\lambda}\right)(\lambda-T-D)-S K \in \Phi_{+}(X)$. Since $S K \in \mathcal{P}(X)$, by Proposition 3 we have $\lambda-T-S-D \in \Phi_{+}(X)$ where $D \in \mathcal{J}_{T}(X)$, hence $\lambda \notin \sigma_{e 1, \varepsilon}(T+S)$. We conclude that $\sigma_{e 1, \varepsilon}(T+S) \subset \sigma_{e 1, \varepsilon}(T)$. 
Theorem 6. Let $T, S \in B \mathcal{R}(X)$. If for every $\lambda \notin \sigma_{e j, \varepsilon}(T)$, where $j \in\{4,5\}$, there exists $A_{\lambda}$ a left inverse modulo compact of $(\lambda-T-D)$ for all $D \in \mathcal{J}_{T}(X)$ such that $S A_{\lambda} \in \Lambda_{T}(X)$ and $\operatorname{dim}\left(S A_{\lambda}(0)\right)<\infty$, then $\sigma_{e 4, \varepsilon}(T+S) \subset \sigma_{e 4, \varepsilon}(T)$.

Proof. Let $\lambda \notin \sigma_{e 4, \varepsilon}(T)$. Then $\lambda-T-D \in \Phi_{+}(X)$ for all $D \in \mathcal{J}_{T}(X)$. Since $A_{\lambda}$ a left inverse modulo compact operator of $(\mu-T-D)$ such that $S A_{\lambda} \in \Lambda_{T}(X)$ and $\operatorname{dim}\left(S A_{\lambda}(0)\right)<\infty$,

$$
\lambda-T-S-D=\left(I-S A_{\lambda}\right)(\lambda-T-D)-S K .
$$

As, $S A_{\lambda} \in \Lambda_{T}(X)$, then by Theorem 3 we get $I-S A_{\lambda} \in \Phi(X)$. By using Lemma 5 we obtain $\left(I-S A_{\lambda}\right)(\lambda-T-D) \in \Phi(X)$. According to Proposition 3, we conclude that $\lambda-T-S-D \in$ $\Phi(X)$ where $D \in \mathcal{J}_{T}(X)$. Then $\lambda \notin \sigma_{e 4, \varepsilon}(T+S)$.

Theorem 7. Let $T, S \in L \mathcal{R}(X)$ and $\lambda-T-S-D \in \Phi_{+}(X)$ for all $D \in \mathcal{J}_{T}(X)$.

$\left(H_{1}\right)$ If there exists $H_{\lambda}$ a left inverse modulo compact of $(\lambda-T-S-D)$ such that $-\lambda^{-1}(T+$ D) $S H_{\lambda}$ is demicompact, then

$$
\left[\sigma_{e 1, \varepsilon}(T+S)\right] \backslash\{0\} \subset\left[\sigma_{e 1, \varepsilon}(T) \cup \sigma_{e 1}(S)\right] \backslash\{0\} .
$$

$\left(H_{2}\right)$ Moreover, if there exists $G_{\lambda}$ a left inverse modulo compact of $(\lambda-T-S-D)$ such that $-\lambda^{-1} S(T+D) G_{\lambda}$ is demicompact, then

$$
\left[\sigma_{e 1, \varepsilon}(T) \cup \sigma_{e 1}(S)\right] \backslash\{0\}=\left[\sigma_{e 1, \varepsilon}(T+S)\right] \backslash\{0\} .
$$

Proof. $(i)$ Let $\lambda \in \mathbb{C} \backslash\{0\}$. If there exists $H_{\lambda}$ a left inverse modulo compact of $(\lambda-T-S-D)$ for all $D \in \mathcal{J}_{T}(X)$, then by Theorem 1 we get

$$
H_{\lambda}(\lambda-T-S-D)=I-K \text { where } K \in \mathcal{K}(X) \text {. }
$$

Since $T \in B \mathcal{R}(X)$ and $D \in \mathcal{J}_{T}(X)$, by Proposition 1 (ii) we have $(\lambda-T-D)(\lambda-S)=\lambda(\lambda-T-D)-(\lambda-T-D) S$. From Lemma 3, it follows that

$$
\begin{gathered}
\lambda(\lambda-T-D)-(\lambda-T-D) S=\lambda(\lambda-T-D)-\lambda S+(T+D) S= \\
=\lambda(\lambda-T-S-D)+(T+D) S .
\end{gathered}
$$

Hence, $(T+D) S K(0)=(T+D) S(0) \subset(\lambda(\lambda-T-S-D)+(T+D) S)(0)$ and $\mathcal{D}(\lambda(\lambda-$ $T-S-D)+(T+D) S)=\mathcal{D}((T+D) S K)$. Then by Lemma 2 we get

$$
(\lambda-T-D)(\lambda-S)=\lambda(\lambda-T-S-D)+(T+D) S+(T+D) S K-(T+D) S K .
$$

Clearly, $\mathcal{D}((T+D) S)=X$, then

$$
\begin{gathered}
(\lambda-T-D)(\lambda-S)=\lambda(\lambda-T-S-D)+(T+D) S(I-K)+(T+D) S K= \\
=\lambda(\lambda-T-S-D)+(T+D) S H_{\lambda}(\lambda-T-S-D)+(T+D) S K .
\end{gathered}
$$

By using Lemma 3, we get $\lambda(\lambda-T-S)+(T+D) S H_{\lambda}(\lambda-T-S-D)+(T+D) S K=$ $\lambda\left(I+\lambda^{-1}(T+D) S H_{\lambda}\right)(\lambda-T-S-D)+(T+D) S K$. We conclude

$$
(\lambda-T-D)(\lambda-S)=\lambda\left(I+\lambda^{-1}(T+D) S H_{\lambda}\right)(\lambda-T-S-D)+(T+D) S K
$$


Since $\mathcal{D}((T+D) S K)=\mathcal{D}\left(\lambda\left(I+\lambda^{-1}(T+D) S H_{\lambda}\right)(\lambda-T-S-D)\right)$ and $(T+D) S K(0) \subset$ $\lambda(\lambda-T-S-D)+(T+D) S(I-K)(0)$, we obtain

$$
(T+D) S K(0) \subset \lambda\left(I+\lambda^{-1}(T+D) S H_{\lambda}\right)(\lambda-T-S-D)(0) .
$$

Using Lemma 2, we prove that

$$
(\lambda-T-D)(\lambda-S)-(T+D) S K=\lambda\left(I+\lambda^{-1}(T+D) S H_{\lambda}\right)(\lambda-T-S-D) .
$$

Let $\lambda \notin\left[\sigma_{e 1, \varepsilon}(T) \cup \sigma_{e 1}(S)\right] \backslash\{0\}$, then $\lambda-T-D \in \Phi_{+}(X)$ for all $D \in \mathcal{J}_{T}(X)$ and $\lambda-$ $S \in \Phi_{+}(X)$. Hence, $(T+D) S K \in \mathcal{P}(X)$. By Lemma 5 (iii) and Proposition 3 we get $(\lambda-T-D)(\lambda-S)-(T+D) S K \in \Phi_{+}(X)$. Again, by using Lemma $5(v)$, we show that $\lambda-T-S-D \in \Phi_{+}(X)$ for all $D \in \mathcal{J}_{T}(X)$. We deduce that, $\lambda \notin\left[\sigma_{e 1, \varepsilon}(T+S)\right] \backslash\{0\}$.

(ii) Continuing in the same way, we can find

$$
(\lambda-S)(\lambda-T-D)=\lambda(\lambda-T-S-D)\left(I+\lambda^{-1} S(T+D) G_{\lambda}\right)+S(T+D) K .
$$

Let $\lambda \notin\left[\sigma_{e 1, \varepsilon}(T+S)\right] \backslash\{0\}$. Then $\lambda-T-S-D \in \Phi_{+}(X)$ for all $D \in \mathcal{J}_{T}(X)$. Since $-\lambda^{-1}(T+D) S H_{\lambda}$ and $-\lambda^{-1} S(T+D) G_{\lambda}$ are demicompact, by Theorem 2 we have $I+$ $\lambda^{-1}(T+D) S H_{\lambda}$ and $I+\lambda^{-1} S(T+D) G_{\lambda}$ are upper semi-Fredholm. Therefore, by Lemma 5 we have $\lambda(\lambda-T-S-D)\left(I+\lambda^{-1}(T+D) S H_{\lambda}\right)$ and $\lambda\left(I+\lambda^{-1}(T+D) S H_{\lambda}\right)(\lambda-T-S-D)+$ $(T+D) S K)$ are upper semi-Fredholm. Consequently, $(T+D) S K, S(T+D) K \in \mathcal{P}(X)$, then $(\lambda-T-D)(\lambda-S)$ and $(\lambda-S)(\lambda-T-D)$ are upper semi-Fredholm. Applying Lemma 5 we conclude $\lambda-T-D \in \Phi_{+}(X)$ for all $D \in \mathcal{J}_{T}(X)$ and $\lambda-S \in \Phi_{+}(X)$. We deduce that $\lambda \notin\left[\sigma_{e 1, \varepsilon}(T) \cup \sigma_{e 1}(S)\right] \backslash\{0\}$.

4. Pseudospectrum of $2 \times 2$ block matrices of multivalued linear operator. In this section we investigate the pseudospectrum of $2 \times 2$ block matrices multivalued linear operator of the form

$$
\mathcal{L}=\left(\begin{array}{ll}
A & B \\
C & D
\end{array}\right)
$$

where the linear relation $A$ acts on $X$ and has the domain $\mathcal{D}(A), D$ is defined on $\mathcal{D}(D)$ and acts on the Banach space $X$, and the intertwining $B$ (resp. $C$ ) is defined on the domain $\mathcal{D}(B)($ resp. $\mathcal{D}(C))$ and acts on $X($ resp. on $X)$.

Remark 1 ([7], Remark 4.1). Let

$$
\mathcal{T}=\left(\begin{array}{cc}
T_{1} & 0 \\
0 & T_{2}
\end{array}\right)
$$

where $T_{1}, T_{2} \in L \mathcal{R}(X)$. Defining the norm of the multivalued linear operator matrix $\mathcal{T}$ as

$$
\|\mathcal{T}\|=\max \left\{\left\|T_{1}\right\|,\left\|T_{2}\right\|\right\} .
$$

Theorem 8 ([7], Theorem 4.1). Let $A, B \in L \mathcal{R}(X)$ and $C \in B \mathcal{R}(X)$. Consider the multivalued linear operator matrix $\mathcal{M}_{C}=\left(\begin{array}{cc}A & C \\ 0 & B\end{array}\right)$. Then for all $\lambda \in \mathbb{C}$ we have:

$$
\lambda-\mathcal{M}_{C}-\mathcal{T}=\left(\begin{array}{cc}
I & 0 \\
0 & \lambda-B-T_{2}
\end{array}\right)\left(\begin{array}{cc}
I & -C \\
0 & I
\end{array}\right)\left(\begin{array}{cc}
\lambda-A-T_{1} & 0 \\
0 & I
\end{array}\right) .
$$


(ii) Let $C_{1}$ be a selection of $C$ and $\mathcal{D}\left[\left(\begin{array}{cc}I & 0 \\ 0 & \lambda-B-T_{2}\end{array}\right)\right]$ containing the ranges of both

$$
\begin{aligned}
\left(\begin{array}{cc}
\lambda-A-T_{1} & -C_{1} \\
0 & I
\end{array}\right) \text { and } & \left(\begin{array}{cc}
0 & C-C \\
0 & 0
\end{array}\right) \text {, then } \\
\lambda-\mathcal{M}_{C}-\mathcal{T} & =\left(\begin{array}{cc}
I & 0 \\
0 & \lambda-B-T_{2}
\end{array}\right)\left(\begin{array}{cc}
I & -C_{1} \\
0 & I
\end{array}\right)\left(\begin{array}{cc}
\lambda-A-T_{1} & 0 \\
0 & I
\end{array}\right)+ \\
& +\left(\begin{array}{cc}
0 & C-C \\
0 & \left(\lambda-B-T_{2}\right)(C-C)
\end{array}\right) .
\end{aligned}
$$

Theorem 9 ([7], Theorem 4.2). (i) If $\left(A+B+T_{1}\right)(0) \subset N(C)$, then for all $\lambda \in \mathbb{C} \backslash\{0\}$ we have

$$
\lambda-\mathcal{L}-\mathcal{T}=-\frac{1}{\lambda}\left(\begin{array}{cc}
0 & 0 \\
C\left(A+T_{1}\right) & C B
\end{array}\right)+\left(\begin{array}{cc}
I & 0 \\
-\frac{1}{\lambda} C & I
\end{array}\right)\left(\begin{array}{cc}
\lambda-A-T_{1} & -B \\
0 & \lambda-D-T_{2}
\end{array}\right) .
$$

(ii) Let $C_{1}$ be a selection of $C$. If $\left(A+B+T_{1}\right)(0) \subset N(C)$, then for all $\lambda \in \mathbb{C} \backslash\{0\}$ we have

$$
\lambda-\mathcal{L}-\mathcal{T}=-\frac{1}{\lambda}\left(\begin{array}{cc}
0 & 0 \\
C\left(A+T_{1}\right) & C B
\end{array}\right)+\left(\begin{array}{cc}
I & 0 \\
-\frac{1}{\lambda} C_{1} & I
\end{array}\right)\left(\begin{array}{cc}
\lambda-A-T_{1} & -B \\
0 & \lambda-D-T_{2}
\end{array}\right) .
$$

(iii) Let $C_{1}$ (resp. $\left.B_{1}\right)$ be a selection of $C$ (resp. B). If $\left(A+B+T_{1}\right)(0) \subset N(C)$, then for all $\lambda \in \mathbb{C} \backslash\{0\}$ we have

$$
\begin{gathered}
\lambda-\mathcal{L}-\mathcal{T}=\left(\begin{array}{cc}
I & 0 \\
-\frac{1}{\lambda} C_{1} & I
\end{array}\right)\left(\begin{array}{cc}
\lambda-A-T_{1} & -B_{1} \\
0 & \lambda-D-T_{2}
\end{array}\right)- \\
-\frac{1}{\lambda}\left(\begin{array}{cc}
0 & \lambda(B-B) \\
C\left(A+T_{1}\right) & C B+C_{1}(B-B)
\end{array}\right) .
\end{gathered}
$$

Theorem 10. Let $A, B, C \in B \mathcal{R}(X), \lambda \in \mathbb{C} \backslash\{0\}$ and $C_{1}$ be a selection of $C$. Suppose that for every $\lambda \notin \sigma_{e 1}(A)$ there exists $A_{\lambda}$ a left inverse modulo compact of $(\lambda-A)$ such that $T_{1} A_{\lambda}$ is demicompact and $\operatorname{dim}\left(T_{1} A_{\lambda}(0)\right)<\infty$ for all $T_{1} \in \mathcal{J}_{T}(X)$. Assume that

$$
\left(\begin{array}{cc}
0 & C-C \\
0 & \left(\lambda-B-T_{2}\right)(C-C)
\end{array}\right) \in \mathcal{P}_{+}(X \times X)
$$

then $\sigma_{e 1, \varepsilon}\left(\mathcal{M}_{C}\right) \subset \sigma_{e 1}(A) \cup \sigma_{e 1, \varepsilon}(B)$.

Proof. Let $T_{1}, T_{2} \in L \mathcal{R}(X)$ and consider $\mathcal{T}=\left(\begin{array}{cc}T_{1} & 0 \\ 0 & T_{2}\end{array}\right)$ such that $\|\mathcal{T}\|=\max \left\{\left\|T_{1}\right\|\right.$, $\left.\left\|T_{2}\right\|\right\}<\varepsilon$ where $T_{1}(0) \subset A(0), T_{2}(0) \subset B(0), \mathcal{D}(A) \subset \mathcal{D}\left(T_{1}\right)$ and $\mathcal{D}(B) \subset \mathcal{D}\left(T_{2}\right)$. By Theorem 8 (ii), we have

$$
\begin{aligned}
\lambda-\mathcal{M}_{C}-\mathcal{T}= & \left(\begin{array}{cc}
I & 0 \\
0 & \lambda-B-T_{2}
\end{array}\right)\left(\begin{array}{cc}
I & -C_{1} \\
0 & I
\end{array}\right)\left(\begin{array}{cc}
\lambda-A-T_{1} & 0 \\
0 & I
\end{array}\right) \\
& +\left(\begin{array}{cc}
0 & C-C \\
0 & \left(\lambda-B-T_{2}\right)(C-C)
\end{array}\right) .
\end{aligned}
$$


By Proposition 2, we have $\lambda-A-T_{1}$ and $\lambda-B-T_{2}$ is closed. Let $\lambda \notin \sigma_{e 1}(A)$. Then $\lambda-A \in \Phi_{+}(X)$. By Theorem 1 , there exists $A_{\lambda} \in \mathcal{B}(X)$ and a finite rank projection $K$ such that $A_{\lambda}(\lambda-A)=I-K$. Since $T_{1}(0) \subset A(0)$ and $\mathcal{D}\left(T_{1} K\right)=\mathcal{D}(A)=X$, by Lemma 2 we have

$$
\lambda-A-T_{1}=\lambda-A-T_{1}+T_{1} K-T_{1} K .
$$

By using Proposition 1 (ii) we get

$$
\lambda-A-T_{1}+T_{1} K-T_{1} K=\lambda-A-T_{1}(I-K)-T_{1} K=\lambda-A-T_{1} A_{\lambda}(\lambda-A)-T_{1} K .
$$

By Lemma 3 we get $\lambda-A-S-T_{1}=\left(I-S A_{\lambda}\right)(\lambda-A)-T_{1} K$. As $S A_{\lambda}$ is demicompact and $\operatorname{dim}\left(T_{1} A_{\lambda}(0)\right)<\infty$, it follows from Theorem 2 that $\left(I-T_{1} A_{\lambda}\right) \in \Phi_{+}(X)$ and we have $(\lambda-A) \in \Phi_{+}(X)$. Then by Theorem 5 we get

$$
\lambda-A-T_{1}=\left(I-T_{1} A_{\lambda}\right)(\lambda-A)-T_{1} K \in \Phi_{+}(X) .
$$

Let $\lambda \notin \sigma_{e 1}(A) \cup \sigma_{e 1, \varepsilon}(B)$ and $\|\mathcal{T}\|=\max \left\{\left\|T_{1}\right\|,\left\|T_{2}\right\|\right\}<\varepsilon$,

$$
\mathcal{T}\left(\begin{array}{l}
0 \\
0
\end{array}\right)=\left(\begin{array}{l}
T_{1}(0) \\
T_{2}(0)
\end{array}\right) \subset\left(\begin{array}{l}
A(0) \\
B(0)
\end{array}\right)=\mathcal{M}_{C}\left(\begin{array}{l}
0 \\
0
\end{array}\right)
$$

$\mathcal{D}\left(\mathcal{M}_{C}\right)=\mathcal{D}(A) \times[\mathcal{D}(C) \cap \mathcal{D}(B)] \subset \mathcal{D}\left(T_{1}\right) \times \mathcal{D}\left(T_{2}\right)=\mathcal{D}(T)$. Therefore $\lambda-A-T_{1} \in \Phi(X)$ and $\lambda-B-T_{2} \in \Phi_{+}\left(X_{2}\right)$. This let us conclude that

$$
\left(\begin{array}{lc}
I & 0 \\
0 & \lambda-B-T_{2}
\end{array}\right),\left(\begin{array}{cc}
\lambda-A-T_{1} & 0 \\
0 & I
\end{array}\right) \in \Phi_{+}(X \times X)
$$

We claim that $\left(\begin{array}{cc}I & -C_{1} \\ 0 & I\end{array}\right)$ is invertible and

$$
\left(\begin{array}{cc}
0 & C-C \\
0 & \left(\lambda-B-T_{2}\right)(C-C)
\end{array}\right) \in \mathcal{P}_{+}(X \times X),
$$

then $\lambda-\mathcal{M}_{C}-\mathcal{T} \in \Phi_{+}\left(X_{\times} X_{2}\right)$ for all $\|\mathcal{T}\|<\varepsilon$

$$
\mathcal{T}\left(\begin{array}{l}
0 \\
0
\end{array}\right)=\left(\begin{array}{l}
T_{1}(0) \\
T_{2}(0)
\end{array}\right) \subset\left(\begin{array}{l}
A(0) \\
B(0)
\end{array}\right)=\mathcal{M}_{C}\left(\begin{array}{l}
0 \\
0
\end{array}\right)
$$

and $\mathcal{D}\left(\mathcal{M}_{C}\right)=\mathcal{D}(A) \times[\mathcal{D}(C) \cap \mathcal{D}(B)] \subset \mathcal{D}\left(T_{1}\right) \times \mathcal{D}\left(T_{2}\right)=\mathcal{D}(\mathcal{T})$. We conclude that $\lambda \notin$ $\sigma_{e 1, \varepsilon}\left(\mathcal{M}_{C}\right)$.

Remark 2. Let $\varepsilon>0$ and $A, B \in C \mathcal{R}(X), C \in \mathcal{L}(X)$. Suppose that for every $\lambda \notin \sigma_{e 1}(A)$ there exists $A_{\lambda}$ a left inverse modulo compact of $(\lambda-A)$ such that $T_{1} A_{\lambda}$ is demicompact and $\operatorname{dim}\left(T_{1} A_{\lambda}(0)\right)<\infty$ for all $T_{1} \in \mathcal{J}_{T}(X)$. We consider the multivalued matrix operator $\mathcal{M}_{C}=\left(\begin{array}{cc}A & C \\ 0 & B\end{array}\right)$. Then we have

$$
\sigma_{e 1, \varepsilon}\left(\mathcal{M}_{C}\right) \subset \sigma_{e 1, \varepsilon}(A) \cup \sigma_{e 1, \varepsilon}(B) .
$$


Theorem 11. Let $A, B, C \in B \mathcal{R}(X), \lambda \in \mathbb{C} \backslash\{0\}$ and $C_{1}$ be a selection of $C$.

(i) Let for every $\lambda \notin \sigma_{e 4}(A)$ there exist $A_{\lambda}$ a left inverse modulo compact of $(\lambda-A)$ such that $T_{1} A_{\lambda} \in \Lambda_{T}(X)$ and $\operatorname{dim}\left(T_{1} A_{\lambda}(0)\right)<\infty$ for all $T_{1} \in \mathcal{J}_{T}(X)$. Assume that

$$
\left(\begin{array}{cc}
0 & C-C \\
0 & \left(\lambda-B-T_{2}\right)(C-C)
\end{array}\right) \in \mathcal{P}_{+}(X \times X),
$$

then $\sigma_{e 4, \varepsilon}\left(\mathcal{M}_{C}\right) \subset \sigma_{e 4}(A) \cup \sigma_{e 4, \varepsilon}(B)$.

(ii) Let for every $\lambda \notin \sigma_{e 5}(A)$ there exist $A_{\lambda}$ a left inverse modulo compact of $(\lambda-A)$ such that $T_{1} A_{\lambda} \in \Lambda_{T}(X)$ and $\operatorname{dim}\left(T_{1} A_{\lambda}(0)\right)<\infty$ for all $T_{1} \in \mathcal{J}_{T}(X)$. Assume that

$$
\left(\begin{array}{cc}
0 & C-C \\
0 & \left(\lambda-B-T_{2}\right)(C-C)
\end{array}\right) \in \mathcal{P}_{+}(X \times X)
$$

and

$$
\operatorname{dim}\left[\left(\begin{array}{cc}
\lambda-A-T_{1} & 0 \\
0 & I
\end{array}\right)\left(\begin{array}{l}
0 \\
0
\end{array}\right) \cap N\left(\left(\begin{array}{cc}
I & 0 \\
0 & \lambda-B-T_{2}
\end{array}\right)\left(\begin{array}{cc}
I & -C_{1} \\
0 & I
\end{array}\right)\right)\right]=0
$$

then

$$
\sigma_{e 5, \varepsilon}\left(\mathcal{M}_{C}\right) \subset \sigma_{e 5}(A) \cup \sigma_{e 5, \varepsilon}(B) .
$$

Proof. ( $i)$ Let $\lambda \notin \sigma_{e 4}(A)$ be such that $\lambda-A \in \Phi_{+}(X)$. Since $A_{\lambda}$ is a left inverse modulo compact operator of $(\mu-A)$ such that $T_{1} A_{\lambda} \in \Lambda_{T}(X)$ and $\operatorname{dim}\left(T_{1} A_{\lambda}(0)\right)<\infty$,

$$
\lambda-A-T_{1}=\left(I-T_{1} A_{\lambda}\right)\left(\lambda-A-T_{1}\right)-T_{1} K .
$$

As, $T_{1} A_{\lambda} \in \Lambda_{T}(X)$, then by Theorem 3 we get $I-T_{1} A_{\lambda} \in \Phi(X)$. By using Lemma 5 we get $\left(I-T_{1} A_{\lambda}\right)(\lambda-A) \in \Phi(X)$. According to Proposition 3, we conclude that $\lambda-A-T_{1} \in \Phi(X)$ where $T_{1} \in \mathcal{J}_{T}(X)$. Let $\lambda \notin \sigma_{e 4}(A) \cup \sigma_{e 4, \varepsilon}(B)$

$$
\|\mathcal{T}\|=\max \left\{\left\|T_{1}\right\|,\left\|T_{2}\right\|\right\}<\varepsilon, \mathcal{T}\left(\begin{array}{l}
0 \\
0
\end{array}\right)=\left(\begin{array}{l}
T_{1}(0) \\
T_{2}(0)
\end{array}\right) \subset\left(\begin{array}{l}
A(0) \\
B(0)
\end{array}\right)=\mathcal{M}_{C}\left(\begin{array}{l}
0 \\
0
\end{array}\right)
$$

and $\mathcal{D}\left(\mathcal{M}_{C}\right)=\mathcal{D}(A) \times[\mathcal{D}(C) \cap \mathcal{D}(B)] \subset \mathcal{D}\left(T_{1}\right) \times \mathcal{D}\left(T_{2}\right)=\mathcal{D}(\mathcal{T})$. Therefore $\lambda-A-T_{1} \in \Phi(X)$ and $\lambda-B-T_{2} \in \Phi\left(X_{2}\right)$. This let us conclude that $\lambda-\mathcal{M}_{C}-\mathcal{T} \in \Phi\left(X_{\times} X_{2}\right)$ for all $\|\mathcal{T}\|<\varepsilon$,

$$
\mathcal{T}\left(\begin{array}{l}
0 \\
0
\end{array}\right)=\left(\begin{array}{l}
T_{1}(0) \\
T_{2}(0)
\end{array}\right) \subset\left(\begin{array}{l}
A(0) \\
B(0)
\end{array}\right)=\mathcal{M}_{C}\left(\begin{array}{l}
0 \\
0
\end{array}\right)
$$

and $\mathcal{D}\left(\mathcal{M}_{C}\right)=\mathcal{D}(A) \times[\mathcal{D}(C) \cap \mathcal{D}(B)] \subset \mathcal{D}\left(T_{1}\right) \times \mathcal{D}\left(T_{2}\right)=\mathcal{D}(\mathcal{T})$. We conclude that

$$
\lambda \notin \sigma_{e 1, \varepsilon}\left(\mathcal{M}_{C}\right) .
$$

(ii) Let $\lambda \notin \sigma_{e 5}(A)$. Then for all $T_{1} \in \mathcal{J}_{T}(X)$ such that $\lambda-A \in \Phi_{+}(X)$ and $i(\lambda-A)=0$. Since $A_{\lambda}$ is a left inverse modulo compact operator of $(\mu-A)$ such that $T_{1} A_{\lambda} \in \Lambda_{T}(X)$ and $\operatorname{dim}\left(T_{1} A_{\lambda}(0)\right)<\infty$, by Theorem 3 we get

$$
I-T_{1} A_{\lambda} \in \Phi(X) \text { and } i\left(I-T_{1} A_{\lambda}\right)=0 .
$$

By using Lemma 5 , we get $\left(I-T_{1} A_{\lambda}\right)(\lambda-A) \in \Phi(X)$ and by Lemma 6 we have $i((I-$ $\left.\left.T_{1} A_{\lambda}\right)(\lambda-A)\right)=0$. According to Proposition 3 , we conclude that $\lambda-A-T_{1} \in \Phi(X)$ and 
$i\left(\lambda-A-T_{1}\right)=0$ where $D \in \mathcal{J}_{T}(X)$.

Let $\lambda \notin \sigma_{e 5}(A) \cup \sigma_{e 5, \varepsilon}(B),\|\mathcal{T}\|=\max \left\{\left\|T_{1}\right\|,\left\|T_{2}\right\|\right\}<\varepsilon$,

$$
\mathcal{T}\left(\begin{array}{l}
0 \\
0
\end{array}\right)=\left(\begin{array}{l}
T_{1}(0) \\
T_{2}(0)
\end{array}\right) \subset\left(\begin{array}{l}
A(0) \\
B(0)
\end{array}\right)=\mathcal{M}_{C}\left(\begin{array}{l}
0 \\
0
\end{array}\right)
$$

and $\mathcal{D}\left(\mathcal{M}_{C}\right)=\mathcal{D}(A) \times[\mathcal{D}(C) \cap \mathcal{D}(B)] \subset \mathcal{D}\left(T_{1}\right) \times \mathcal{D}\left(T_{2}\right)=\mathcal{D}(\mathcal{T})$. Therefore $\lambda-A \in \Phi(X)$, $i(\lambda-A)=0$ and $\lambda-B-T_{2} \in \Phi(X), i\left(\lambda-B-T_{2}\right)=0$. This let us conclude that

$$
\lambda-\mathcal{M}_{C}-\mathcal{T} \in \Phi(X \times X)
$$

for all $\|\mathcal{T}\|<\varepsilon, \mathcal{T}\left(\begin{array}{l}0 \\ 0\end{array}\right)=\left(\begin{array}{l}T_{1}(0) \\ T_{2}(0)\end{array}\right) \subset\left(\begin{array}{l}A(0) \\ B(0)\end{array}\right)=\mathcal{M}_{C}\left(\begin{array}{l}0 \\ 0\end{array}\right)$ and $\mathcal{D}\left(\mathcal{M}_{C}\right)=\mathcal{D}(A) \times[\mathcal{D}(C) \cap$ $\mathcal{D}(B)] \subset \mathcal{D}\left(T_{1}\right) \times \mathcal{D}\left(T_{2}\right)=\mathcal{D}(\mathcal{T})$. Hence, $i\left(\lambda-A-T_{1}\right)=0$, then $i\left[\left(\begin{array}{cc}\lambda-A-T_{1} & 0 \\ 0 & I\end{array}\right)\right]=0$ and $i\left[\left(\begin{array}{cc}I & 0 \\ 0 & \lambda-B-T_{2}\end{array}\right)\right]=0$. By using Proposition 3, we get

$$
i\left(\mathcal{M}_{C}\right)=i\left(\left(\begin{array}{cc}
\lambda-A-T_{1} & 0 \\
0 & I
\end{array}\right)\left(\begin{array}{cc}
I & 0 \\
0 & \lambda-B-T_{2}
\end{array}\right)\left(\begin{array}{cc}
I & -C_{1} \\
0 & I
\end{array}\right)\right) .
$$

By applying Lemma 6, we obtain

$$
\begin{gathered}
i\left(\lambda-\mathcal{M}_{C}-\mathcal{T}\right)=i\left(\left(\begin{array}{cc}
\lambda-A-T_{1} & 0 \\
0 & I
\end{array}\right)\right)+i\left(\left(\begin{array}{cc}
I & 0 \\
0 & \lambda-B-T_{2}
\end{array}\right)\right)\left(\begin{array}{cc}
I & -C_{1} \\
0 & I
\end{array}\right)+ \\
+\operatorname{dim}\left[\left(\begin{array}{cc}
\lambda-A-T_{1} & 0 \\
0 & I
\end{array}\right)\left(\begin{array}{l}
0 \\
0
\end{array}\right) \cap N\left(\left(\begin{array}{cc}
I & 0 \\
0 & \lambda-B-T_{2}
\end{array}\right)\left(\begin{array}{cc}
I & -C_{1} \\
0 & I
\end{array}\right)\right)\right], \\
=i\left(\left(\left(\begin{array}{cc}
I & 0 \\
0 & \lambda-B-T_{2}
\end{array}\right)\right)+i\left(\left(\begin{array}{cc}
I & -C_{1} \\
0 & I
\end{array}\right)\right)+\right. \\
+\operatorname{dim}\left[N\left(\left(\begin{array}{cc}
I & 0 \\
0 & \lambda-B-T_{2}
\end{array}\right)\right) \cap\left(\left(\begin{array}{cc}
I & -C_{1} \\
0 & I
\end{array}\right)\left(\begin{array}{l}
0 \\
0
\end{array}\right)\right) .\right.
\end{gathered}
$$

Since $C_{1}$ is single valued, we get

$$
\left(\begin{array}{cc}
I & -C_{1} \\
0 & I
\end{array}\right)\left(\begin{array}{l}
0 \\
0
\end{array}\right)=\left(\begin{array}{l}
0 \\
0
\end{array}\right)
$$

We claim that $\left(\begin{array}{cc}I & -C_{1} \\ 0 & I\end{array}\right)$ is invertible, then $i\left(\left(\begin{array}{cc}I & -C_{1} \\ 0 & I\end{array}\right)\right)=0$. Therefore,

$$
i\left(\lambda-\mathcal{M}_{C}-\mathcal{T}\right)=0
$$

It follows immediately from (1) and (2) that

$$
\sigma_{e 5, \varepsilon}\left(\mathcal{M}_{C}\right) \subset \sigma_{e 5}(A) \cup \sigma_{e 5, \varepsilon}(B) .
$$

Remark 3. Let $\varepsilon>0$ and $A, B \in B \mathcal{R}(X), C \in \mathcal{L}(X)$.

(i) Let for every $\lambda \notin \sigma_{e 5}(A)$ there exist $A_{\lambda}$ a left inverse modulo compact of $(\lambda-A)$ such that $T_{1} A_{\lambda} \in \Lambda_{T}(X)$ and $\operatorname{dim}\left(T_{1} A_{\lambda}(0)\right)<\infty$ for all $T_{1} \in \mathcal{J}_{T}(X)$. We consider the multivalued matrix operator $\mathcal{M}_{C}=\left(\begin{array}{cc}A & C \\ 0 & B\end{array}\right)$, then we have

$$
\sigma_{e 4, \varepsilon}\left(\mathcal{M}_{C}\right) \subset \sigma_{e 4}(A) \cup \sigma_{e 4, \varepsilon}(B) .
$$


(ii) Let for every $\lambda \notin \sigma_{e 5}(A)$ there exist $A_{\lambda}$ a left inverse modulo compact of $(\lambda-A)$ such that $T_{1} A_{\lambda} \in \Lambda_{T}(X)$ and $\operatorname{dim}\left(T_{1} A_{\lambda}(0)\right)<\infty$ for all $T_{1} \in \mathcal{J}_{T}(X)$. Assume that

$$
\operatorname{dim}\left[\left(\begin{array}{cc}
\lambda-A-T_{1} & 0 \\
0 & I
\end{array}\right)\left(\begin{array}{l}
0 \\
0
\end{array}\right) \cap N\left(\left(\begin{array}{cc}
I & 0 \\
0 & \lambda-B-T_{2}
\end{array}\right)\left(\begin{array}{cc}
I & -C \\
0 & I
\end{array}\right)\right)\right]=0,
$$

then

$$
\sigma_{e 5, \varepsilon}\left(\mathcal{M}_{C}\right) \subset \sigma_{e 5}(A) \cup \sigma_{e 5, \varepsilon}(B) .
$$

Theorem 12. Let $C_{1}$ be a selection of $C$ and $\left(A+B+T_{1}\right)(0) \subset N(C)$. Then for all $\lambda \in \mathbb{C} \backslash\{0\}$. Let for every $\lambda \notin \sigma_{e 1}(A)$ there exist $A_{\lambda}$ a left inverse modulo compact of $(\lambda-A)$ such that $T_{1} A_{\lambda}$ is demicompact and $\operatorname{dim}\left(T_{1} A_{\lambda}(0)\right)<\infty$ for all $T_{1} \in \mathcal{J}_{T}(X)$. Assume that

$$
\left(\begin{array}{cc}
0 & \lambda(B-B) \\
C\left(A+T_{1}\right) & C B+C_{1}(B-B)
\end{array}\right) \in \mathcal{P}_{+}(X \times X)
$$

then

$$
\sigma_{e 1, \varepsilon}(\mathcal{L}) \backslash\{0\} \subset\left[\sigma_{e 1}(A) \cup \sigma_{e 1, \varepsilon}(D)\right] \backslash\{0\} .
$$

Proof. Let $\mathcal{T}=\left(\begin{array}{cc}T_{1} & 0 \\ 0 & T_{2}\end{array}\right)$ such that $\|\mathcal{T}\|<\varepsilon$,

$$
\mathcal{T}\left(\begin{array}{l}
0 \\
0
\end{array}\right)=\left(\begin{array}{l}
T_{1}(0) \\
T_{2}(0)
\end{array}\right) \subset\left(\begin{array}{l}
A(0) \\
B(0)
\end{array}\right)=\left(\begin{array}{cc}
A & B_{1} \\
0 & D
\end{array}\right)\left(\begin{array}{l}
0 \\
0
\end{array}\right)
$$

and

$$
\mathcal{D}\left(\left(\begin{array}{cc}
A & B_{1} \\
0 & D
\end{array}\right)\right)=\mathcal{D}(A) \times\left[\mathcal{D}\left(B_{1}\right) \cap \mathcal{D}(D)\right] \subset \mathcal{D}\left(T_{1}\right) \times \mathcal{D}\left(T_{2}\right)=\mathcal{D}(\mathcal{T})
$$

If $\lambda \notin \sigma_{e 1}(A) \cup \sigma_{e 1, \varepsilon}(D) \cup\{0\}$, then by Remark 2 we get

$$
\lambda \notin \sigma_{e 1, \varepsilon}\left[\left(\begin{array}{cc}
A & B_{1} \\
0 & D
\end{array}\right)\right] .
$$

We infer that $\lambda-\left(\begin{array}{cc}A & B_{1} \\ 0 & D\end{array}\right)-\mathcal{T} \in \Phi_{+}(X \times X)$ for all $\|\mathcal{T}\|<\varepsilon$,

$$
\mathcal{T}\left(\begin{array}{l}
0 \\
0
\end{array}\right)=\left(\begin{array}{cc}
A & B_{1} \\
0 & D
\end{array}\right)\left(\begin{array}{l}
0 \\
0
\end{array}\right)
$$

and $\mathcal{D}\left(\left(\begin{array}{cc}A & B_{1} \\ 0 & D\end{array}\right)\right)=\mathcal{D}(\mathcal{T})$. This is equivalent to

$$
\left(\begin{array}{cc}
\lambda-A-T_{1} & -B_{1} \\
0 & \lambda-D-T_{2}
\end{array}\right) \in \Phi_{+}(X \times X) .
$$

Using the fact that

$$
\left(\begin{array}{cc}
0 & \lambda(B-B) \\
C\left(A+T_{1}\right) & C B+C_{1}(B-B)
\end{array}\right) \in \mathcal{P}_{+}(X \times X)
$$


we ensure that

$$
\left(\begin{array}{cc}
\lambda-A-T_{1} & -B_{1} \\
0 & \lambda-D-T_{2}
\end{array}\right)+\left(\begin{array}{cc}
0 & \lambda(B-B) \\
C\left(A+T_{1}\right) & C B+C_{1}(B-B)
\end{array}\right) \in \Phi_{+}(X \times X) .
$$

By using Lemma 9 (iii), we get $\lambda-\mathcal{L}-\mathcal{T} \in \Phi_{+}(X \times X)$ for all $\|\mathcal{T}\|<\varepsilon$,

$$
\mathcal{T}\left(\begin{array}{l}
0 \\
0
\end{array}\right)=\left(\begin{array}{cc}
A & B_{1} \\
0 & D
\end{array}\right)\left(\begin{array}{l}
0 \\
0
\end{array}\right), \quad \mathcal{D}\left(\left(\begin{array}{cc}
A & B_{1} \\
0 & D
\end{array}\right)\right)=\mathcal{D}(\mathcal{T}) .
$$

This let us conclude that

$$
\lambda \notin \sigma_{e 1, \varepsilon}(\mathcal{L}) .
$$

\section{REFERENCES}

1. A. Jeribi, Spectral theory and applications of linear operator and block operator matrices, SpringerVerlag, New York, 2015.

2. F. Abdmouleh, A. Jeribi, T. Alvarez, On a characterization of the essential spectra of a linear relation, 2012, preprint.

3. T. Alvarez, A. Ammar, A. Jeribi, A characterization of some subsets of S-essential spectra of a multivalued linear operator, Colloq. Math., 135 (2014), 171-186.

4. T. Alvarez, R.W. Cross, D. Wilcox, Multivalued Fredholm type operators with abstract generalised inverses, J. Math. Anal. Appl., 261 (2001), №1, 403-417.

5. T. Alvarez, A. Ammar, A. Jeribi, On the essential spectra of some matrix of lineair relations, Math. Methods Appl. Sci., 37 (2014) 620-644.

6. A. Ammar, A. Jeribi, B. Saadaoui, Frobenius-Schur factorization for multivalued $2 \times 2$ matrices linear operator, Mediterr. J. Math., 14 (2017), №1, 14-29.

7. A. Ammar, A. Jeribi, B. Saadaoui, A characterization of essential pseudospectra of the multivalued operator matrix, Anal. Math. Phys., DOI 10.1007/s13324-017-0170-z.

8. A. Ammar, A. Jeribi, B. Saadaoui, Demicompactness, Slection of linear relation and application to multivalued matrix, Preprint, 2018.

9. A. Ammar, H. Daoud, A. Jeribi, Pseudospectra and essential pseudospectra of multivalued linear relations, Mediterr. J. Math. 12 (2015), no. 4, 1377-1395.

10. A. Ammar, H. Daoud, A. Jeribi, The stability of pseudospectra and essential pseudospectra of linear relation, J. Pseudo-Differ. Oper. Appl., 7 (2016), №4, 473-491.

11. W.Chaker, A. Jeribi, B. Krichen, Demicompact linear operators, essential spectrum and some perturbation results, Math. Nachr., 288 (2015), №13, 1476-1486.

12. E. Chafai, M. Mnif, Perturbation of normally solvable linear relations in paracomplete spaces, Linear Algebra Appl., 439 (2013), №7, 1875-1885.

13. R.W. Cross, Multivalued linear operators, Marcel Dekker, 1998.

14. R.W. Cross, An index theorem for the product of linear relations, Linear Algebra Appl., 277 (1998), $127-134$.

15. E.B. Davies, Linear Operators and their spectra, Cambridge Studies in Advanced Mathematics, 106, Camb. Univ. Press, Cambridge, 2007.

16. B. Krichen, Relative essential spectra involving relative demicompact unbounded linear operators, Acta Math. Sci. Ser. B Engl. Ed., 34 (2014), №2, 546-556.

17. H.J. Landau, On Szegö's eigenvalue distribution theorem and non-Hermitian kernels, J. Anal. Math., 28 (1975), 335-357.

18. W.V. Petryshyn, Construction of fixed points of demicompact mappings in Hilbert space, J. Math. Anal. Appl., 14 (1966), 276-284. 
19. L.N. Trefethen, Pseudospectra of matrices, numerical analysis, 1991, In Pitman Research notes in Mathematics Series, Longman Science and Technology, Harlow 260, 1992, 234-266.

20. L.N. Trefethen, Pseudospectra of linear operators, SAIM rev., 39 (1997), 383-406.

21. J.M. Varah, The computation of bounds for the invariant subspaces of a general matrix operator, Ph.D. thesis, stanford university ProQuest LLC, Ann Arbor, 1967.

22. J.H. Wilkinson, Sensitivity of eigenvalues II, Util. Math., 30 (1986), 243-286.

Departement of Mathematics

University of Sfax

Faculty of Sciences of Sfax

Tunisia

ammar_aymen84@yahoo.fr

Aref.Jeribi@fss.rnu.tn

saadaoui.bilel@hotmail.fr 